\title{
GLOWing future for fracture prediction tools?
}

Taking comorbidities into account when calculating fracture risk in woman aged $\geq 55$ years can improve fracture prediction, indicate results of a recent study in Bone. A higher comorbidity index, defined as the number of comorbidities at baseline, was associated with a greater risk of sustaining fractures. The researchers also found that the FRAX $^{\circledR}$ algorithm was a more reliable predictor of fracture incidence when the comorbidity index was taken into account during risk calculation.

The researchers gathered data on 52,960 women from 10 countries who participated in the Global Longitudinal Study of Osteoporosis in Women (GLOW). The women self-reported their medical history when starting the study and were contacted annually to find out if they had sustained a fracture in the intervening year. The total follow-up period was 2 years.

Of the 13 comorbidities assessed, Parkinson disease, multiple sclerosis, chronic obstructive pulmonary disease, osteoarthritis and heart disease were associated with an increased risk of fracture, but not high cholesterol levels, hypertension or cancer. Parkinson disease was the comorbidity most significantly associated with increased fracture risk (age-adjusted HR 2.2; 95\% CI 1.6-3.1).

All data in the study were collected using a self-reporting questionnaire. Although this approach is limited by factors such as recall bias, the survey format would make reporting of comorbidites and fractures standardized, thus limiting the variability that can be introduced when extracting data from medical records and databases in different countries. To apply this method in a clinical setting would also be fairly simple. However, the classification of comorbidities would need to be carefully evaluated and precise definitions set out before this information could be used to determine fracture risk. "Further research is now needed to consider inclusion of information of this type into fracture prediction tools," concludes lead researcher Elaine Dennison (University of Southampton).

Bo Abrahamsen (University of Southern Denmark), who was not involved in the study, highlights important issues that need clarification. "The association between

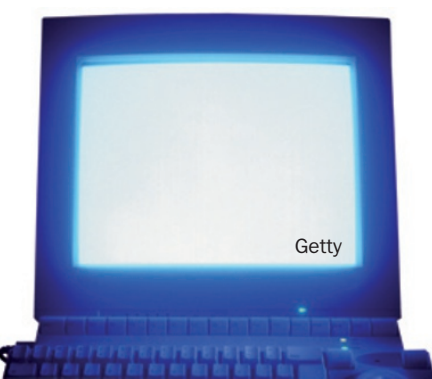

osteoarthritis and fracture risk will come as a surprise to many clinicians, but, as pointed out by the authors, bone loss appears to be accelerated in patients with osteoarthritis. It is also possible, however, that some patients who were considered as osteoarthritis cases had rheumatoid arthritis; the criterion for accepting selfreported rheumatoid arthritis in the study was fairly strict." Abrahamsen also points out that it would have been possible to split the GLOW study, which is very large, into one cohort for deriving risk weights and one cohort for validation, and emphasizes the usefulness of "a validation study within a subsample of GLOW to assess how accurately self-reported conditions tally with patient notes".

Fiona Mitchell

Original article Dennison, E. M. et al. Effect of co-morbidities on fracture risk: findings from the Global Longitudinal Study of Osteoporosis in Women (GLOW). Bone doi:10.1016/j.bone.2012.02.639 